\title{
Las categorías de sentido, sentido personal y sentido subjetivo en una perspectiva histórico-cultural: un camino hacia una nueva definición de subjetividad*
}

\author{
Sense, Personal Sense and Subjective Sense in a Historical-Cultural \\ Perspective: a Path Towards a New Definition of Subjectivity
}

Recibido: marzo 29 de 2009 ｜Ｒevisado: julio 10 de 2009 | Aceptado: julio 17 de 2009

\author{
FERNANDO LUIS GONZÁLEZ-REY***
}

Universidad de Brasilia, Brasil

Artículo de reflexión teórica.

** Departamento de Psicología Clinica, Campus Universitario Darcy Ribeiro, Brasilia, Distrito FederalBrasil 70910-900.

Correo electrónico: gonzalez-rey49@hotmail.com

\section{RES UMEN}

El presente artículo discute una de las categorías centrales desarrolladas por Vygotsky en el último periodo de su obra: el sentido. Se analiza el desarrollo progresivo y acelerado de esa categoría en sus últimos trabajos, así como lo que ella representa en términos de una nueva unidad para el análisis de la psique. Se discuten también algunas de las razones por las cuales esa categoría no encontró un espacio en la Psicología soviética, así como las diferencias entre las categorías de sentido y de sentido personal propuesta por A. N. Leontiev. El uso de la categoría sentido se ha extendido progresivamente en la literatura psicológica en los últimos tiempos, y una discusión sobre este concepto y sus orígenes requiere de una urgente elaboración teórica para evitar la banalización del concepto. Partiendo del concepto de sentido en Vygotsky, en este artículo se propone el concepto de sentido subjetivo como piedra angular para el estudio de la subjetividad desde una visión histórico-cultural.

Palabras claves autor

Sentido, sentido subjetivo, personalidad y subjetividad.

Palabras clave descriptor

Subjetividad, sentidos, personalidad.

\section{A B S T R A C T}

This paper discusses one of the central problems of the last period of the Vygotsky's work: the sense. It is analyzed the progressive and fast development of this category in his last works, as well as its consequences in terms of what it represents as a possible unity of analysis of psyche. Moreover it is discussed some of the reasons by which this category did not find an space in the soviet psychology, as well as the differences between sense and the concept of personal sense introduced by A. N. Leontiev. The use of the category of sense has progressively extended in the psychological literature in the last time and a discussion about the concept and its origins is in urgent need of theoretical elaboration in order to avoid the triviality of the concept. Starting from the concept of sense in Vygotsky I propose the concept of subjective sense as the cornerstone for the development of the topic of the subjectivity from an historical-cultural viewpoint.

Key words author

Sense, Subjective Sense, Personality, Subjectivity.

Key words plus

Subjectivity, Senses, Personality. 


\section{Introducción}

De forma reciente, la categoría de sentido ha tenido una amplia profusión en la literatura psicológica. No obstante ser una categoría general usada por la lingüística donde el término tuvo su origen, la filosofía, el análisis del discurso y la sociología, por solo mencionar algunas ramas del saber donde aparece, no representa un significado único, usándose con frecuencia para designar cuestiones diferentes. Sin embargo, la forma en que el sentido fue empleado en la semiótica rusa -básicamente en los trabajos de Bakhtin- expresa una compatibilidad con el rechazo a la metafísica presente en las obras de Nietzsche y Heidegger, toda vez que el sentido existe en relación, sin quedar cristalizado nunca en un significado susceptible de descripción universal a través de la medida.

Las categorías representan instrumentos del pensamiento que toman significados diferentes en el proceso de su utilización, como se ha evidenciado en muchos casos de la Psicología. Por tanto, el presente trabajo no tiene la pretensión de descalificar algunos de los empleos de la categoría sentido para reivindicar otros, sino el de esclarecer la forma en que ésta evolucionó en la Psicología de orientación histórico-cultural, después de su efímero uso por Vygotsky, con el objetivo de evitar su banalización por un uso indiscriminado en la literatura actual.

Como he expresado en trabajos anteriores (González Rey, 2001, 2002, 2004, 2005, 2008) la categoría de sentido en la perspectiva históricocultural, fue introducida por Vygotsky en el último momento de su obra. Sin embargo, fue ignorada tanto dentro de la Psicología soviética como en la Psicología occidental, pues en ambas las interpretaciones dominantes sobre el autor, partían de referentes poco sensibles al tipo de consecuencias, que una categoría como la de sentido, podía tener para la Psicología.

En la Psicología occidental, apareció identificada con el carácter singular del significado, interpretación muy influida por el sesgo lingüístico y cognitivo de la aproximación sociocultural norteamericana. A pesar de las diferencias, la mayoría de autores identificados con este enfoque, se centraron en una interpretación focalizada en los conceptos de acción y de mediación semiótica, ignorando otras contribuciones muy importantes de su obra (Wertsch, 1997). De este modo, dieron por absolutas las ideas de un momento específico de la obra vygotskiana (1928-1931), desconociendo otros, lo cual ha llevado a una interpretación unilateral, como justamente han señalado Van der Veer y Valsiner (1994):

Está claro que la recepción de las ideas de Vygotsky en Occidente ha sido selectiva. En un sentido esto es inevitable y puede incluso ser productivo; nosotros todos creamos nuestro propio Freud, Piaget o Vygotsky y desarrollamos nuestras ideas sobre nuestros propios "insights". El caso de Vygotsky, sin embargo, es ligeramente diferente porque su trabajo no ha estado de forma general disponible en inglés y (consecuentemente) una aproximación sobria de su trabajo no parece haber sido realizada aún. (p. 6)

En este artículo se intenta aclarar la evolución de la categoría de sentido en la obra de Vygotsky y presentar su evolución en la Psicología soviética, así como sus diferencias con la categoría de sentido subjetivo propuesta en el presente estudio, y que hemos venido desarrollando como base para el tema de la subjetividad en una perspectiva histórico-cultural.

\section{El concepto de sentido en la obra de Vygotsky}

Como ya dijimos, dicho concepto aparece solo en el último período de la obra de Vygotsky y su presencia fue efímera. Sin embargo, fue una idea que en poco tiempo tuvo una interesante evolución. En Pensamiento y Lenguaje (1987), el autor asume este concepto a partir de los trabajos del psicólogo francés Paulhan, quien se centró en el estudio de la relación entre el significado y el sentido de las palabras en el uso del lenguaje. En dicha obra expresa: 
Paulhan afirma que el sentido de la palabra es complejo, fluido y está en cambio permanente. De alguna manera él es único para cada conciencia y para una conciencia individual en circunstancias diferentes. En ese aspecto, el sentido de la palabra es inagotable. La palabra adquiere sentido en una frase. La frase en si misma adquiere sentido, sin embargo, en el contexto del párrafo, el párrafo en el contexto del libro, y el libro en el contexto de los trabajos escogidos del autor. Finalmente, el sentido de la palabra es determinado por todo lo que en la conciencia está relacionado con aquello que se expresa en la palabra. (p. 276)

Algo que Vygotsky destaca en esa obra, sobre todo en su primer y último capítulos, es la significación de los aspectos procesales de la relación entre pensamiento, palabra y lenguaje, así como la relación de esas funciones con la conciencia como sistema. En ese enfoque, expresa la tendencia general de su pensamiento en la comprensión de la psique como sistema (que tiene su base en unidades que reproducen en sí mismas las características dinámicas del todo). En aquel momento avanzado de su trabajo, parecía orientado al desarrollo de un nuevo tipo de unidad de la vida psíquica. Esta interpretación encuentra apoyo en la siguiente afirmación hecha por Luria (1987) en el epílogo de las Obras Escogidas de Vygotsky:

No es solo el sentido que está más allá de la palabra. El sentido no es el elemento final de esta cadena. Más allá de la palabra están los afectos y las emociones. Sin la exploración de las relaciones de la palabra con el motivo, la emoción y la personalidad, el análisis del problema de "Pensamiento y Lenguaje" queda incompleto. (p. 369)

Sin duda, ese análisis quedó incompleto, pero no solo en relación con el sentido, sino en su propia representación sobre la relación del pensamiento con la personalidad. Esas fueron cuestiones que Vygotsky nos dejó abiertas para la interpretación de su legado y que hasta hoy no han sido desarrolladas en el análisis de su obra. Como hemos defendido en trabajos anteriores (González Rey, 2007, 2008), su producción fue rica, contradictoria y en constante desarrollo, pero no maduró en algunas de sus tesis esenciales, entre otras cosas, por su muerte prematura. Su trabajo se vio también atravesado por la tensión entre su visión de la psique como reflejo y la defensa de su carácter generador. Esta óptica era dominante en la Psicología soviética y llevaba implícito el peso de su connotación ideológica, pues en ella descansaba el carácter materialista del psiquismo humano.

Las contradicciones del pensamiento vygotskiano se evidencian a lo largo de toda su obra. Fue, como la mayoría de los clásicos, un pensamiento inquieto, vivo, en movimiento. En la última etapa de su trabajo, entre 1932 y 1934, simultáneamente con la categoría de sentido, también usó la de vivencia - Psicología de la Edad (1932) y El problema del ambiente (1934) ${ }^{1}$ — definida como la categoría psicológica capaz de dar cuenta de la unidad de lo cognitivo y lo afectivo en el desarrollo humano.

$\mathrm{Al}$ igual que la categoría sentido, el concepto de vivencia fue efímero, no apareciendo en otras obras del mismo período en las que su uso hubiera representado un avance en el desarrollo general de su sistema de pensamiento. El hecho de que las categorías de vivencia y sentido hayan coexistido en el tiempo, y que Vygotsky no haya profundizado la relación entre ellas, ni su alcance para explicar la relación de lo cognitivo y lo afectivo en otras funciones psíquicas como la imaginación, tema al que dedicó una de sus Lecturas de Psicología en 1932, nos indica que, posiblemente, no tuvo la intención teórica que le atribuimos en relación con esas categorías.

Un análisis muy interesante sobre las limitaciones que Vygotsky tuvo para representarse la unidad de lo cognitivo y lo afectivo a través de la categoría de vivencia, fue realizado por su discípula L. I. Bozhovich (1981), quien escribió:

1 El problema del ambiente fue el nombre que Van Der Veer y Valsiner atribuyeron a ese texto que formó parte de sus conferencias en la Facultad de Paidología del Instituto Estadual Pedagógico Hertzen y que fueron publicadas como Bases de la Paidología en 1935, por ese mismo instituto, un año después de su muerte. El título de esa conferencia, definido por los autores, se debe a que las conferencias solo estaban numeradas, siendo ese título el de la conferencia número 6. 
Vygotsky comenzó a buscar la correspondiente "unidad" [se refiere a la unidad de lo cognitivo y lo afectivo] en el estudio de la "situación social del desarrollo". Como tal distinguió la vivencia... Y si el concepto de vivencia planteado por él, nos acercó a la interpretación de las verdaderas causas del desarrollo infantil, la búsqueda posterior del eslabón que determina ese desarrollo, búsqueda que termina en el concepto de generalización, de nuevo nos hizo regresar a posiciones intelectualistas. (pp. 123-125)

Vygotsky atribuyó la vivencia a la capacidad de generalización del niño, con lo cual no pudo avanzar de forma consecuente en su propuesta de una categoría psíquica capaz de expresar la unidad de lo cognitivo y lo afectivo en el desarrollo humano. El principio de reflejo tuvo, entre sus consecuencias, la orientación dominante de la Psicología soviética en el estudio de las funciones cognitivas que se podían representar sobre la base de la información procedente del mundo, evitando la espinosa cuestión del carácter generador de la psique.

Una vez más coincidimos con Luria (1987) cuando expresa:

Vygotsky no fue capaz de explorar todas esas cuestiones en detalle [se está refiriendo a las cuestiones señaladas por él en la cita anterior que usamos de su trabajo]. Es importante enfatizar, sin embargo, que la relación entre sentido y significado, y la relación entre lo intelectual y lo afectivo, fueron los focos de gran parte de sus trabajos en los últimos años de su vida. (p. 369)

Es indudable que Vygotsky, como Luria señala, se centró tanto en la última parte de su obra como en la primera - pasada por alto no solo por Luria sino por todos los integrantes del grupo de Jarkov-, en la compleja relación entre cognición y afecto. El hecho de que, como hemos dicho ya antes, las categorías nunca representaran contenidos analíticos parciales, sino momentos de un sistema teórico en desarrollo, nos lleva a pensar que con la categoría sentido se empeñaba en desarrollar una nueva representación sobre la mente en una perspectiva histórico-cultural.
En sus primeras referencias, Vygotsky se mantuvo en la categoría sentido dentro de los mismos límites de Paulhan, asociándola esencialmente al uso de la palabra y a su relación con las estructuras de significado del lenguaje. Sin embargo, y dentro del propio texto "Pensamiento y palabra", último capítulo de Pensamiento y Lenguaje, enfatiza cada vez más la relación del sentido con la personalidad y con la vida psíquica como un todo.

Así, en una de las formas más acabadas en que consigue definir la categoría de sentido en esa obra, escribe (1987):

El sentido de una palabra es el agregado de todos los elementos psicológicos que aparecen en nuestra conciencia como resultado de la palabra. El sentido es una formación dinámica, fluida y compleja que tiene varias zonas que varían en su estabilidad. El significado es apenas una de esas zonas del sentido que la palabra adquiere en el contexto del habla. El es el más estable, unificado y preciso de esas zonas. (p. 276)

En la cita se evidencia un atributo importante de esa definición: el hecho de que él considere al sentido como una formación, lo que apunta a la integración de esa categoría en el sistema psíquico, no limitándola a su relación con la palabra. Sin embargo, esto es una inferencia que no queda explícita en su obra. El intento de presentar la compleja articulación entre pensamiento, lenguaje, habla, personalidad y conciencia, como sistema en movimiento, abre una nueva opción para entender lo mental que no se apoya en el análisis de las partes, sino en la comprensión dinámica de la psique como sistema. El lenguaje es un elemento recursivo permanente del desarrollo del sistema y no algo externo a él.

Esa visión integradora y compleja sobre la psique se expresa también en el primer capítulo de Pensamiento y Lenguaje cuando expresa (1987):

El aspecto de sentido del habla, de hecho, el aspecto interno completo del habla que está orientado a la personalidad no ha sido hasta tiempos muy recientes un territorio de la Psicología (...) El resultado ha 
sido que las relaciones entre pensamiento y palabra se han comprendido como constantes, relaciones eternas entre cosas, no como relaciones internas, dinámicas y móviles entre procesos. (p. 283)

Algo sorprendente en el autor fue su extraordinaria capacidad para articular cuestiones que históricamente habían sido analizadas de forma parcial y estática, dentro de sistemas en movimiento, en los cuales las diferentes funciones psíquicas adquieren significado para la Psicología. Pienso que esta fue una de las razones principales que lo llevó a interesarse por la categoría personalidad, en cuyo uso veía una posibilidad de integración de las diferentes funciones psíquicas, como sistema psíquico.

La representación de la psique humana ha sido uno de los aspectos más omitidos por las diferentes formas de apropiación del pensamiento vygotskiano en Occidente, el cual con frecuencia se presenta disperso a través de un conjunto de categorías concretas, sin destacar la articulación de esas categorías con el sistema psíquico, como fue propuesto en cada uno de los momentos de su obra. Incluso los diferentes momentos de su producción solo han sido objeto de análisis hasta épocas muy recientes (González Rey, 2008; Leontiev, 1992; Van der Veer \& Valsiner, 1990). Ese desconocimiento llevó a omitir lo que quizás fue su mayor contribución: la refundación de una representación general de la psique humana en las condiciones de su desarrollo cultural.

En otra cita tomada del primer capítulo de Pensamiento y Lenguaje, el autor señala (1987):

La primera cuestión que emerge cuando consideramos la relación entre pensamiento y lenguaje y los otros aspectos de la vida de la conciencia, tiene que ver con la conexión entre intelecto y afecto. Entre los mayores defectos básicos de las aproximaciones tradicionales al estudio de la Psicología ha estado el aislamiento del aspecto intelectual de los aspectos volitivos y afectivos de la conciencia. La inevitable consecuencia del aislamiento de estas funciones ha sido la transformación del pensamiento en una corriente autónoma. El pensamiento se transforma a sí mismo en el pensador de pensamientos. El pensamiento fue divorciado de la completa vitalidad de la vida, de los motivos, intereses e inclinaciones del pensamiento individual. (p. 50)

En esa cita aparece su orientación hacia la comprensión de las funciones psíquicas en términos del sujeto concreto. Insiste en la necesidad de considerar el pensamiento en su integración inseparable con los procesos afectivos de la persona. En múltiples ocasiones expresó que esa unidad era la base del sistema psicológico. En ese sentido, afirma (1987):

La dirección en que nos debemos mover en nuestro intento de resolver este problema vital [se refiere a esa compleja conexión entre el pensamiento, el afecto y el comportamiento] está indicada por el método que confía en el análisis del todo complejo en sus unidades. Existe un "sistema dinámico de significados" que constituye una unidad de los procesos afectivos e intelectuales. Toda idea contiene alguna reminiscencia de las relaciones afectivas individuales de aquel aspecto de la realidad que ella representa. De esa manera, el análisis en unidades permite ver la relación entre las necesidades individuales o inclinaciones y el pensamiento. (pp. 50-51)

Al presentar ese "sistema dinámico de significados", que integra al pensamiento, el comportamiento y la personalidad en un sistema complejo y dinámico, Vygotsky vuelve a destacar la necesidad de un nuevo tipo de unidad para la comprensión de la psique capaz de integrar la cognición y el afecto en una relación procesal y no estática. Algo que ha llamado mucho nuestra atención es que la misma cita que acabamos de referir, tomada de la edición de sus Obras Escogidas en inglés, en que aparece el concepto "sistema dinámico de significados", la usa A. A. Leontiev (1992) en traducción directa del ruso al inglés y, en lugar de "sistema dinámico de significados" aparece el concepto de "sistema dinámico de sentidos", lo cual tiene una enorme trascendencia para nuestras reflexiones sobre el alcance de la categoría sentido. Leontiev señala: 
De forma general no es la unidad de lo cognitivo y lo afectivo como tal, sino la realización de esa unidad en forma de un "sistema dinámico de sentidos", el cual relaciona la "dinámica del pensamiento" [intelecto] así como la "dinámica del comportamiento y la actividad concreta de la personalidad" (Tomado de Vygotsky, 1982, p. 22 en ruso). (p. 42)

En caso de que la cita original en ruso se refiera a "sistema dinámico de sentidos" se tendrían que revisar muchas otras de las interpretaciones en la Psicología actual, y eso ayudaría en la definición del verdadero alcance de la categoría sentido y de las extraordinarias consecuencias que pudiera tener para el desarrollo de la Psicología actual.

Otra cuestión interesante en el trabajo citado de Leontiev, es que, a pesar de haber sido uno de los teóricos importantes de la Psicología soviética y rusa del siglo XX, el artículo referido antes tuvo poca repercusión, tanto en Rusia como en Occidente, lo que puede explicarse por el hecho de que va en un sentido completamente diferente al que tomaron las interpretaciones sobre Vygotsky, tanto en las posiciones dominantes de la Psicología soviética, como en la Psicología norteamericana.

En la Psicología soviética, los trabajos sobre el sentido tuvieron un resurgimiento en la década de los ochenta del siglo XX, particularmente orientados por la categoría de sentido personal definida por A. N. Leontiev ${ }^{2}$ en los años cuarenta, poco después de la muerte de Vygotsky. En la definición no hay ninguna referencia a la forma en que Vygotsky la había desarrollado; el resurgimiento se debió a algunos de sus discípulos más jóvenes como Bratus, Asmolov, Stolin, Subotsky, entre otros, interesados en el desarrollo del tema de la personalidad insuficientemente tratado en el marco de la teoría de la actividad. Esos trabajos se orientaron por algunas modificaciones realizadas por el propio A. N. Leontiev en Actividad, Conciencia y Personalidad, una de sus últimas publicaciones, en la que intentó

2 A. N. Leontiev fue el padre de A. A. Leontiev, ambos referidos en el presente trabajo, pero que para el lector que no conozca esta información, le puede pasar desapercibido que la única diferencia entre ambos está en la letra que diferencia el patronímico. separarse de sus posiciones más radicalmente reduccionistas y deterministas de momentos anteriores. En ese esfuerzo, el autor dejó de definir el sentido personal como la relación entre los motivos y los objetivos de la actividad, para definirlo como relación entre motivos.

Continuando con el análisis de la categoría de sentido en los últimos trabajos de Vygotsky, nos deparamos con su artículo Sobre la cuestión de la Psicología de la creatividad del actor (1984). En ese trabajo, publicado en ruso en 1932, afirma:

En el proceso de la vida societal (...) las emociones entran en nuevas relaciones con otros elementos de la vida psíquica, nuevos sistemas aparecen, nuevos conjuntos de funciones psíquicas; unidades de un orden superior emergen, gobernadas por leyes especiales, dependencias mutuas, y formas especiales de conexión y movimiento. (p. 328)

En relación con esta cita, que también usa en el artículo antes referido, A. A. Leontiev comenta: "Precisamente ese conjunto es la unidad de 'cognición y afecto'. iÉl forma el sistema dinámico de sentidos!" (p. 42). Nuevamente, legitima el uso del término sistema dinámico de sentidos, por parte de Vygotsky. Creo que esa afirmación es la consecuencia necesaria y posible de vislumbrar un nuevo camino en aquel momento de la obra, sin embargo, como expresé antes, no creo que el pensador tuviera conciencia del alcance teórico de la categoría de sentido.

Esta última cita representó, posiblemente, el momento más evolucionado del pensamiento vygotskiano. En ella el autor consigue algo esencial para la refundación del tema de la subjetividad en una perspectiva histórico-cultural: le atribuye un carácter generador a la psique y reconoce la autonomía relativa de las emociones en los espacios de producción psicológica que, sin duda, apuntan a la conversión del sentido en un nuevo tipo de unidad psicológica. Fue ese el último momento en su reflexión sobre la categoría sentido.

Comoresultado del análisis que Vygotsky nos presenta en Pensamiento y Lenguaje, es posible concluir que sentido y significado son procesos diferentes, 
capaces de articularse de diversas formas en el funcionamiento psíquico y que uno es irreducible al otro. Los significados, aunque con frecuencia representen sentidos en sí mismos, no expresan una relación directa, lineal, ni intencional con los sentidos. En nuestros trabajos hemos enfatizado esa relación, asumiendo el pensamiento no como una función cognitiva, sino como una función de sentido del sujeto (González Rey, 2002, 2004, 2008).

\section{La categoría de sentido en la Psicología Soviética}

El último momento de la obra de Vygotsky fue prácticamente ignorado en la Psicología Soviética, que siempre estuvo dividida en diferentes posiciones teóricas, entre las que se destacaban quienes pretendieron monopolizar su legado, el grupo de Jarkov, liderado por A. N. Leontiev y agrupados en torno a la teoría de la actividad Luria, Zaporochets, Galperin, Bozhovich y Elkonin, entre otros, ${ }^{3}$ y las escuelas agrupadas en torno a Rubinstein, Uznadze y Ananiev. Estos autores, aunque tuvieron importantes coincidencias con Vygotsky en una serie de cuestiones generales de esa Psicología, también tuvieron diferencias, en particular Rubinstein (1964) y Ananiev (1977) quienes criticaron el concepto de interiorización.

Sin embargo, la interpretación de Vygotsky siempre apareció sesgada por las ideas de A. N. Leontiev y el grupo de Jarkov que él encabezó, quienes monopolizaron su legado en la Psicología soviética. Ese grupo solo legitimó aquellos aspectos que, a su vez, los legitimaban a ellos, con lo cual la primera y última etapas de su pensamiento fueron subvaloradas. La primera etapa, sobre todo su libro Psicología del Arte, fue presentada como la obra de un autor todavía inmaduro. Ese curso histórico

3 L. I. Bozhovich muy temprano rompe con el grupo de Jarkov creando su propio grupo en el Instituto de Psicología General y Pedagógica de Moscú, a partir de sus discrepancias con Leontiev en relación con la definición de motivo. Bozhovich fue la única entre los seguidores de Vygotsky en usar los principios de su obra para el desarrollo de los temas de la motivación y la personalidad en la Psicología soviética. puede estar en la base del desconocimiento integral de la obra, tanto en la Psicología soviética, como en la internacional.

Como expresó el filósofo ruso F. Mikjailov (2006), estudioso del pensamiento de Vygotsky, y quien tuvo una estrecha relación con algunos de los representantes más importantes de la teoría de la actividad:

Aquí nosotros tenemos una lógica de autodesarrollo de la vida no una reacción al estímulo externo [se está refiriendo al segundo momento referido por mí sobre la obra de Vygotsky, aquel comprendido entre 1928 y 1931]. No es por gusto que hoy nadie conoce de forma completa y pormenorizada todos los trabajos de Vygotsky [Yo enfatizo: itodos!]. Y yo puedo demostrar a mis pocos lectores la sorprendentemente profunda literatura filosófica del autor. Pero esto no guarda ninguna relación con el mito de la Psicología cultural-histórica. (p. 41)

Ese desconocimiento de la obra fue, en gran parte, el responsable del hecho de que las críticas orientadas contra la teoría de la actividad se dirigieran también a Vygotsky, lo que dificultaba aún más la aproximación de otros autores a su sistema de pensamiento. Solo L. I. Bozhovich destacó, antes de la década de los años ochenta, las diferencias entre la teoría de la actividad y la teoría históricocultural. Posteriormente, esa diferencia fue extensa y explícitamente analizada por Zinchenko (1997).

La asociación que se menciona entre Vygotsky y Leontiev, dominante en la Psicología soviética hasta tiempos recientes, se explicita claramente por A. Abuljanova (1980) cuando expresa: "En el enfoque de Vygotsky y Leontiev en la base de lo psicológico se considera apenas una cualidad, deducida del principio de la actividad y, por eso, todo el edificio de la Psicología se apoya en un solo punto" (p. 57).

Como se puede observar, la crítica va dirigida al momento de Vygotsky más próximo de Leontiev que se caracteriza por el énfasis en la interiorización, la mediación semiótica y el concepto de funciones psíquicas superiores (de 1928 a 1931), definido como teoría cultural-histórica (González 
Rey, 2008; Leontiev, 1992, 2001; Van der Veer \& Valsiner, 1994). Sin embargo, los momentos en que el autor reconoce el carácter generador de la psique, tanto en la primera como en la última parte de su trabajo, destacando la importancia de la unidad de lo cognitivo y lo emocional y los conceptos de personalidad y motivación, está más próximo a Rubinstein y Ananiev, que a Leontiev y el grupo de Jarkov.

\section{El sentido personal en la teoría de la actividad de A. N. Leontiev y el ocultamiento de la categoría de sentido en la obra de Vygotsky}

El desarrollo de la categoría de sentido personal en la obra de A. N. Leontiev fue definido como momento de la actividad y no como una unidad de la vida psíquica, tal como fue comprendido por Vygotsky. En el curso de su obra, Leontiev definió el sentido personal como la relación entre el motivo y los objetivos de la actividad. En Actividad Conciencia y Personalidad, su último libro, publicado unos años antes de su muerte, pasa a enfatizar en su definición la relación de los motivos entre sí, orientándose a la definición de un sistema de motivos de naturaleza psíquica que no tiene relaciones inmediatas con los objetos externos, algo inexistente en su obra anterior. En esa nueva aproximación a la categoría, señala (1981):

...algunos motivos ocupan un cierto lugar hegemónico y subordinan a ellos otros motivos. Otros, por el contrario descienden a la categoría de subordinados e inclusive pierden por completo la función de conferir sentido. La génesis de este movimiento expresa en sí la génesis del sistema de vínculos entre los sentidos personales: la génesis de la personalidad. (p. 173)

Este intento por reconocer un funcionamiento psíquico de naturaleza subjetiva, una producción que deja de tener una relación inmediata y de determinación con lo externo, se vuelve a expresar con nitidez en otro momento de la mencionada obra, cuando afirma (1981):
La personalidad de este modo, deja de ser el resultado de la superposición directa de las influencias externas y se manifiesta como aquello que el hombre hace de sí mismo, como confirmación de su vida humana. Él confirma su vida dentro del quehacer cotidiano, en la comunicación con las demás personas. (p. 183)

En la cita anterior, el autor no solo apunta a una independencia relativa de la personalidad en relación con las influencias externas, sino que destaca una categoría totalmente ignorada por él en momentos anteriores de su obra: la comunicación, la que un tiempo después será reivindicada en su especificidad e importancia en la génesis y el desarrollo psíquico, por autores como Bodaliev, Lomov y Abuljanova, entre otros.

Las limitaciones de la categoría de sentido personal para trabajar en las cuestiones más complejas de la motivación y la personalidad, se evidencian en los propios seguidores de Leontiev que trabajaron esos temas, e intentaron, de una forma u otra, superarlas. En algunos de esos autores las limitaciones de la definición aparecen de forma explícita, como lo demuestra el siguiente fragmento escrito por Asmolov (1984):

El sentido personal representa el reflejo individualizado del mundo, que incluye la relación de la personalidad con aquellos objetos a través de los cuales se desarrolla su actividad y su comunicación. Las más diversas manifestaciones de la cultura, y más ampliamente de las relaciones sociales, asimiladas por el sujeto en el proceso de interiorización de las normas sociales, los conceptos, roles, valores e ideas percibidos por él en los actos y acciones de otras personas, pueden adquirir para él sentido personal, transformándose en "significados para mî". (p. 63)

En la cita aparece una síntesis de todos los defectos de la definición de sentido personal de Leontiev. Entre ellos deseo destacar los siguientes: en primer lugar, la comprensión del sentido personal como reflejo de las relaciones de la personalidad con los objetos, aspecto que no aparecía en la 
definición de sentido de Vygotsky, y que nos revela la tendencia a la "objetualización" que hegemonizó la Teoría de la Actividad; en segundo lugar, el sentido personal se define como resultado de la asimilación, no como producción ni como creación; y, por último, los sentidos personales son resultado de la interiorización, representando un "significado para mí”. En un acto de enorme valor personal e histórico, sin precedentes en la Psicología rusa pues fue posterior a la desaparición de la Unión Soviética, A. A. Leontiev (1992), escribió:

Muchas de las ideas teóricas en esos trabajos [se refiere a los trabajos de la última etapa de la vida de Vygotsky, entre 1933 y 1934] sin embargo, no fueron asumidas por el grupo de Jarkov, o fueron solo parcialmente aceptadas. Ellas fueron apenas percibidas por los historiadores de Vygotsky y fueron deliberadamente ignoradas por sus críticos. La más importante de esas ideas fue la idea de "sentido" o de "campo de sentido". (p. 41)

El propio hijo de A. N. Leontiev reconoce que la categoría sentido no fue asumida por el grupo de Jarkov, liderado por su padre. De aquí es posible deducir que para A. A. Leontiev la categoría de sentido personal no representaba una continuidad de la categoría sentido, tal como fue formulada por Vygotky. En un artículo publicado por uno de aquellos jóvenes discípulos de Leontiev y por el autor del presente artículo, Bratus y González Rey (1982), se afirma:

Evidentemente es necesario separar el concepto de sentido de los límites de una actividad aislada, como había sido definido el concepto de sentido personal en un primer momento por A. N. Leontiev, y convertirlo en una categoría más universal y generalizada, que incluya en sí misma, tanto los casos del sentido personal como comprendido por Leontiev, así como otras de las relaciones de sentido más complejas. Para designar esa categoría fue propuesto el término de formaciones de sentido de la personalidad. (p. 56)

Aunque ya teníamos conciencia de las limitaciones de la categoría de sentido personal para el desarrollo de los temas complejos de la personalidad, el uso del sentido no representaba todavía una alternativa en relación a las categoría de motivo, concepto que históricamente ha respondido a una visión fragmentada, analítica y determinista de la psique, donde el motivo aparece como una "pieza" más del conjunto de elementos que caracterizan la psique. Las formaciones de sentido las definimos como complejas relaciones entre motivos, precisamente buscando su lugar dentro del sistema complejo de la personalidad, superando de esa forma su reducción a los términos de la actividad.

Sin embargo, el uso de la expresión "formaciones del sentido de la personalidad" tuvo declaraciones muy diferentes dentro de aquel grupo de jóvenes psicólogos, lo que evidenciaba su dificultad para superar el imaginario teórico institucionalizado en la obra de A. N. Leontiev. Las barreras de algunos de aquellos autores para comprender el carácter subjetivo de esas formaciones del sentido, venían de la fuerza que mantenía la visión más "objetivista", orientada a comprender el vínculo directo e inmediato de las formaciones del sentido y la actividad. Esas dificultades se expresan claramente en la siguiente cita de Asmolov (1984).

La determinación de los sistemas dinámicos de sentido [término empleado con frecuencia como sinónimo de formaciones de sentido] por la posición social y considerando el condicionamiento de esta posición a la actividad del sujeto, determina otras particularidades de su naturaleza psicológica: la mediación de los cambios de los sistemas dinámicos de sentido por las transformaciones en la actividad que se encuentran en su base [el principio de la mediación por la actividad de los sistemas de sentido de a personalidad]". (p. 64)

Se percibe, en la reflexión de Asmolov, la dependencia directa, condicionada por la actividad, que establece entre los sistemas dinámicos de sentido y la posición social del sujeto. La actividad está siempre en relación directa con los sistemas dinámicos de sentido.

A partir de aquellos años, a pesar del reconocimiento de la necesidad de ir más allá de los límites 
de la categoría de sentido personal, lo que fue una de las razones de la emergencia de la categoría de formación de sentido, esos intentos fueron tomando diferentes matices, sin llegar a una definición que permitiera un camino alternativo para la Psicología en términos ontológicos, epistemológicos y de sus prácticas, que es lo que se puede vislumbrar de la forma en que Vygotsky usó la categoría de sentido.

Algunos psicólogos soviéticos, aunque conocedores de que se estaba inaugurando una producción teórica que permitía subvertir los cimientos más estables de la Psicología en aquel momento, no lograron cambiar los principios generales dominantes en la representación de la Psicología soviética.

En la evolución de nuestras ideas ante el desafío que implicaba la construcción de nuevas unidades psíquicas capaces de expresar, sin reminiscencias metafísicas, un orden en que lo psíquico apareciera como producción asociada a las prácticas sociales del hombre, sin que se redujera a ellas, lo cual continuaría atrapando un proceso en otro volviendo a una posición metafísica, introdujimos la categoría de sentido subjetivo en un intento por superar las limitaciones que se percibían en la categoría de sentido vygotskiana. El sentido subjetivo representaba una producción subjetiva, en la que lo simbólicoy lo emocional se integran en un proceso recursivo que expresa el valor subjetivo de ciertas definiciones de la cultura, asociadas con diferentes prácticas humanas. Avanzando en esa dirección, en 1995 desarrollamos el concepto de configuración subjetiva, y se pasó a usar el término sentido psicológico para especificar algo que no nos quedaba muy claro todavía a nivel teórico. Sobre esos temas se expresó (1995):

En el momento actual, en el enfoque configuracional de la personalidad, entiendo por configuración la integración de múltiples elementos de la personalidad alrededor de un sentido psicológico específico, por lo que se puede incluir una configuración dentro de otra, no como elementos diferentes que se integran, sino como parte de un nuevo nivel cualitativo de organización psíquica. (p. 59)
En ese momento de nuestro trabajo, sin especificar la naturaleza diferenciada de esa nueva unidad psicológica que seguía en proceso de gestación, ya se atribuyó a determinadas combinaciones de elementos psíquicos, sentidos psicológicos diferenciados, lo que superaba la tendencia a definir necesidades y motivos a partir de contenidos estáticos y universales de los que se esperaba una respuesta congruente en el comportamiento. El sentido psicológico implicó una nueva dimensión en la representación de lo psíquico orientada a especificar su especificidad ontológica ${ }^{4}$ en las condiciones de la cultura, definición que fue precursora del sentido subjetivo.

\section{Del sentido psicológico al sentido subjetivo: el rescate de la cuestión de la subjetividad en una perspectiva histórico-cultural}

A pesar de que el desarrollo de la categoría sentido subjetivo ha sido el centro de nuestro trabajo en los últimos diez años, todavía se percibe que las categorías de sentido y sentido subjetivo son usadas indistintamente y que, el poco rigor en el conocimiento sobre los orígenes de ambas en el marco histórico-cultural, puede llevar a su banalización.

Con la conciencia de las limitaciones de la teoría de la actividad, de la cual fuimos críticos desde los primeros trabajos, y de la relevancia de la categoría sentido en Vygotsky, se coincide con A. A. Leontiev (1992) cuando sostuvo que esa categoría llevaba no solo a una nueva comprensión de su

4 Por el uso frecuente del término ontología en el presente trabajo, se quiere enfatizar que el uso del término se refiere a la naturaleza diferenciada de diferentes problemas del conocimiento humano que encuentran su especificidad en las representaciones teóricas y epistemológicas de las diferentes ciencias. O sea, que la especificidad de aquello que destaco como ontológicamente diferente, no se refiere a una naturaleza universal del ser, como ocurrió con el uso del término en la metafísica, sino a la especificidad de la representación teórica de formas diferentes de lo real que solo son inteligibles en sus representaciones conceptuales y que no expresan un reflejo de cómo ese real aparece fuera de sus relaciones con el conocimiento. Haciendo esto, se intenta superar el relativismo radical que separa conocimiento y realidad de forma igualmente metafísica. 
trabajo, sino a nuevos desdoblamientos y líneas de trabajo en la Psicología. En la profundización sobre la categoría sentido, fuimos comprendiendo su significación, para el desarrollo del tema de la subjetividad, sobre nuevas bases teóricas y epistemológicas.

Cuando se introdujo la categoría de sentido subjetivo (González Rey, 1995), nos propusimos superar la reducción del sentido subjetivo a la palabra, y se definió como la expresión simbólicoemocional de la realidad en sus múltiples efectos, directos y colaterales, sobre la organización subjetiva actual del sujeto y de los espacios sociales en que aquel actúa.

No creemos que lo que A. A. Leontiev identificó como el "verdadero sistema de sentidos" haya sido claro para la representación de Vygotsky, en aquel tiempo. Se desarrolló la definición de sentido subjetivo con el objetivo de especificar nuestras diferencias en relación con la categoría de sentido, tal como fue definida por el autor. Entre las diferencias que se quieren destacar están:

- Mientras el sentido enfatiza la relación entre lo cognitivo y lo emocional, el sentido subjetivo enfatiza la relación entre lo simbólico y emocional, incluyendo todas las formas de producción simbólica. La relación entre lo aspectos simbólico-emocionales que expresa la inmersión del sujeto en el mundo, está en desarrollo permanente y tiene múltiples desdoblamientos, independizándose de los elementos originales que le dieron origen.

- El sentido subjetivo es inseparable de la subjetividad como sistema, pues en cada momento de producción de sentido subjetivo ocurre una integración tensa, múltiple y contradictoria, entre las configuraciones subjetivas presentes del sujeto y en desarrollo en el curso de su acción, y la multiplicidad de efectos colaterales que, resultantes de esa acción, se asocian a nuevas producciones de sentido subjetivo.

En el camino de desarrollo del sentido subjetivo se partió del concepto de configuración subjetiva, cuya primera definición fue formulada como sigue (1995):

Nuestra definición de sentido subjetivo se orienta a presentar el sentido como momento constituido y constituyente de la subjetividad, como aspecto definidor de ésta, en cuanto es capaz de integrar diferentes formas de registro (social, biológico, ecológico, semiótico, etc.) en una organización subjetiva que se define por la articulación compleja de emociones, procesos simbólicos y significados, que toma formas variables y que es susceptible de aparecer en cada momento con una determinada forma de organización dominante. (p. 18)

En esa definición ya se presentaba un elemento esencial del sentido subjetivo; la forma en que aparecen en ellos los registros diferenciados de la experiencia vivida, los cuales pasan a organizarse en otro nivel, el subjetivo. Con esa posición pretendíamos separarnos de cualquier tipo de relación directa y lineal entre esa producción psicológica y cualquiera de los procesos y eventos implicados en su génesis, lo que se considera un aspecto esencial en la especificidad ontológica de lo subjetivo. Definimos lo subjetivo no por oposición a lo objetivo, lo que sería una versión de subjetivismo, sino como representación de un tipo diferente de objetividad, específica de los procesos psíquicos humanos en las condiciones de la cultura.

En el 2002, se avanzó en esa definición y se definió el sentido subjetivo como "la relación inseparable entre lo simbólico y lo emocional, donde uno evoca al otro sin ser su causa" (p. 147). El concepto de sentido subjetivo está en la base de una propuesta sobre la subjetividad en una perspectiva histórico-cultural. Este camino, orientado al desarrollo del tema de la subjetividad en una perspectiva dialógica y compleja, es congruente con el desarrollo de la Psicología soviética, donde la intención de comprender la articulación de la cultura y la personalidad fue un tema recurrente.

La subjetividad como sistema de configuraciones subjetivas que se organiza en las prácticas de individuos y grupos, expresando la tensión entre 
su organización inicial, y las formas que toma en el curso de esas prácticas, tiene una fuerte inspiración interdisciplinar representando una dimensión de todos los procesos humanos. La subjetividad no es la introducción de un concepto, sino una nueva matriz teórica capaz de generar nuevas inteligibilidades múltiples sobre problemas que solo son viables desde su definición teórica. Unido a eso, el tema lleva a importantes cambios en la comprensión de los conceptos psicológicos tradicionales.

Los sentidos subjetivos y las configuraciones subjetivas, son producciones que tienen lugar en el curso de la vida social y la cultura, pero que no están determinados ni por una ni por la otra, no son un reflejo de esos múltiples procesos, sino una nueva producción que los específica en sus efectos para quienes los viven. Como muy bien expresó Vygotsky (1993) en Defecto y Compensación, escrito en 1924 y publicado en 1925:

Ellos no comprendieron [se refiere a los psicólogos que trabajaban el tema en la época] que el 'handicap' no representa solo el empobrecimiento de un estado psicológico, sino una fuente de bienestar, no solo una debilidad, sino una fortaleza. Ellos piensan que el desarrollo del ciego se centra en su ceguera. La psicología del ciego es esencialmente la psicología de la victoria sobre la ceguera". (p. 57)

La definición anterior en relación a la psicología del ciego es una expresión del carácter generador atribuido por Vygotsky a la psique humana, en la primera parte de su obra.

El sentido subjetivo y las configuraciones subjetivas son importantes no solo porque permiten comprender la acción individual en su carácter sistémico, sino porque nos permite entender la sociedad en una nueva dimensión: en su sistema de consecuencias sobre el hombre y sobre la organización de sus diferentes espacios de vida social. El vaciamiento de la dimensión subjetiva que preconizan algunos de los representantes del construccionismo social, como Gergen, lejos de reforzar la dimensión social en el análisis de los problemas humanos, la debilita al privarla de uno sus aspectos esenciales: la subjetividad de los grupos, personas e instituciones que configuran la sociedad en sus expresiones y formas vivas de relación.

La subjetividad abre un nuevo camino no solo para la comprensión de la psique, sino para la comprensión de la relación entre individuo y sociedad, y para una definición de la psique como sistema, donde sus diferentes funciones se presenten como momentos del mismo.

\section{Algunas reflexiones finales}

- El tema del sentido en la perspectiva históricocultural fue introducido por Vygotsky, pero no lo pudo desarrollar de forma consecuente, manteniéndose abierto en sus consecuencias para la Psicología. En su obra, el sentido no representa una categoría más, sino una posibilidad para el desarrollo de una nueva unidad psicológica que quedó inconclusa en su propia definición.

- El sentido fue inseparable de la tendencia general de la Psicología soviética a comprender la personalidad como sistema que se forma y desarrolla en la vida social de la persona dentro de ámbitos culturales diferenciados. Los temas de la acción y el lenguaje, siendo centrales en la teoría vygotskiana y ocupando lugares diferentes a lo largo de ésta, nunca estuvieron separados de la intención de una teoría de la mente de carácter histórico-cultural.

- El desarrollo de la categoría sentido subjetivo representa un intento de desarrollar consecuencias y opciones que la definición de sentido de Vygotsky no permitió, entre ellas, el desarrollo de una teoría de la subjetividad en una perspectiva histórico-cultural.

- La primera y última parte de la obra de Vygotsky han permanecido, hasta hoy, poco estudiadas, tanto por la Psicología soviética y rusa, como por la Psicología occidental. El predominio de la teoría de la actividad en la Psicología soviética representó un impedimento para la comprensión de la obra de Vygotsky como sistema. 


\section{Referencias}

Abuljanova, K. A. (1980). Deyatelnosti e psikjologia lichnosti [Actividad y Psicología de la Personalidad]. Moscou: Izdatelstva Nauka [Ciencia].

Ananiev, B. (1977). O problemax sobremennovo Chelabekaznaniya [Sobre los problemas contemporáneos del conocimiento del hombre]. Moscú: Editorial Nauya [Ciencia].

Asmolov, A. G. (1984). Lichnost kak predmet psikjologicheskovo isledavaniya [A personalidade como objeto da investigação psicológica]. Moscú: Izdatelstva Moskoskovo Universiteta, Editorial de la Universidad de Moscú.

Bodaliev, A. A (1983). Lichnost i obchenie [Personalidad y comunicación]. Moscú: Editora pedagógica.

Bozhovich, L. I. (1981). La personalidad y su formación en la edad infantil. La Habana: Editora Habana, Pueblo y Educación.

González Rey, F. (1995). Personalidad, comunicación y desarrollo. La Habana: Editora Habana, Pueblo y Educación.

González Rey, F. (2002). Sujeto y subjetividad: una aproximación histórico-cultural. México. DF: Thomson.

González Rey, F. (2004). O social na Psicología e a psicologia social: a emergência do sujeito. Petrópolis: VOZES.

González Rey, F. (2007). Social and individual subjectivity from an historical cultural standpoint. Critical Social Studies, 9(2), 3-14.

González Rey, F. (2008, ). Different periods in Vygotsky's work: Their implications for arguments regarding his legacy. Personal presentation in the symposium Discussing Vygotsky's Legacy: New alternatives in the understanding of his legacy and unfolding of his work. ISCAR, San Diego, California, Estados Unidos.

Leontiev, A. A. (1992). Ecce Homo. Methodological Problems. Multidisciplinary Newsletter for Activity Theory, 11/12, 41-44.

Leontiev, A. N. (1978). Actividad, conciencia y personalidad. Buenos Aires: Ediciones Ciencias del Hombre.

Leontiev, A. N. (1981). Actividad, conciencia y personalidad. La Habana: Editora Habana, Pueblo y Educación.
Luria, A. (1987). Afterword to the Russian Edition. In R. Rieber \& A. Carton (Eds.), The collected works of L. S. Vygotsky (Vol. I, pp. 359-374). New York: Plenum Press.

Mikjailov, F. (2006). Problems of the method of culturalhistorical psychology. Journal of Russian and European Psychology, 44(1), 21-54.

Rubinstein, S. L. (1964). El Desarrollo de la Psicología. Principios y Métodos. La Habana: Editora del Consejo Nacional de Universidades.

Touraine, A. (1999). Poderemos viver juntos: iguais ou diferentes. Petrópolis: VOZES.

Van der Veer, R. \& Valsiner, J. (1994). In R. Van der Veer \& J. Valsiner (Orgs.), Introduction to The Vygotsky Reader (pp. 1-9). Oxford: Blackwell.

Vygotsky, L. S. (1968). Pensamiento y Lenguaje. Buenos Aires: Ediciones Ciencias del Hombre.

Vygotsky, L. S. (1984). K voprocy o psikhologii tvorchestva aktera [Sobre as questões da psicologia do ator criativo]. Sobranye sochinenya, 6, 320-346.

Vygotsky, L. S. (1987). Thinking and Speech. In R. Rieber \& A. Carton (Eds.), The collected works of L. S. Vygotsky (Vol. I, pp. 43-285). New York: Plenum Press.

Vygotsky, L. S. (1993). Defect and Compensation. In R. Rieber \& A. Carton (Eds.), The collected works of L. S. Vygotsky (Vol. II, pp. 52-64). New York: Plenum Press.

Vygotsky, L. S. (1994). The problem of environment. In R. Van der Veer \& J. Valsiner (Orgs.), Introduction to The Vygotsky Reader (pp. 355-370). Oxford: Blackwell.

Wertsch, J. (1997). La necesidad de la acción en la investigación sociocultural. En J. Wertsch, P. del Rio. \& A. Álvarez (Orgs.), La mente sociocultural (pp. 49. 62). Madrid: Fundación Infancia y Aprendizaje.

Zinchenko, V. (1997). La Psicología sociocultural y la teoría psicológica de la actividad: revisión y proyección hacia el futuro. En J. Wertsch, P. del Rio. \& A. Álvarez (Orgs.), La mente sociocultural. Aproximaciones teóricas y aplicadas (pp. 35-48) Madrid: Fundación Infancia y Aprendizaje. 
